\title{
The Electronic Structures and Optical Properties in Nitrogen-Doped $\mathrm{SnO}_{2}$
}

\author{
Dan-Xu XING, Pei-Ji WANG, and Chang-Wen ZHANG*
}

School of Physics and Technology, University of Jinan, Jinan, Shandong, 250022, People's

Republic of China

ss_wangpj@ujn.edu.cn, zhchwsd@163.com

Keywords: Electronic structure; First-principles calculation

\begin{abstract}
We perform first-principles calculations to study the electronic structures and optical properties of rutile $\mathrm{SnN}_{x} \mathrm{O}_{2-x}$ as a possible photocatalytic material. It is found that $\mathrm{N}$ incorporation lead to the enhancement of $p$ states in the energy gap and the decrease of band gap. The optical transition between $\mathrm{N} p$ and $\mathrm{O} p$ enhances and shifts to high energy range with increasing $\mathrm{N}$ concentrations. The calculations for the optical properties also predict a possible optimum $\mathrm{N}$-doping level in $\mathrm{SnO}_{2}$ with a high visible photoresponse and as a photocatalytic material.
\end{abstract}

\section{Introduction}

Tin dioxide $\left(\mathrm{SnO}_{2}\right)$ is an effective photocatalyst for the remediation of organic pollutants and promising for the possible application to the solar energy conversion [1-5]. Unfortunately, due to its larger wide band gap of $3.6 \mathrm{eV}$, it can absorb only ultraviolet light, which limits its some possible applications in photocatalyst. Therefore, for the sake of utilizing the wider range of solar light, it is necessary to enhance the optical absorption in the visible range.

It has been demonstrated that this problem can be solved by the appropriate ion dopants. Experimently, $\mathrm{Sb}$ and $\mathrm{F}$-doped $\mathrm{SnO}_{2}$ thin films has been widely used in transparent electrodes in solar cells, flat plane displays, and gas sensors. However, Nb doping may induce the some $\mathrm{O}$ vacancies, and its thermal stability has also been challenged [6]. Therefore, it is essential to attempt the other doping ions for overcoming their disadvantages. Furthermore, the $p$ - type $\mathrm{SnO}_{2}$ has been obtained by doping $\mathrm{Al}$, In, or Li [7-9]. Recently, nitrogen has also been proposed to be a good p-type dopant source in experiments [10-13]. In the present work, based on first-principles calculations, the electronic structures and optical properties of $\mathrm{N}$-doped 72-atom systems are investigated, and we predict that $\mathrm{N}$ substitution for $\mathrm{O}$ is responsible for the red-shift phenomenon, as well as the optimal substitutional concentrations.

\section{Computational Details}

The simulations are performed using the full-potential linearized augmented plane-wave method embedded in WIEN2K code [14] within the framework of the generalized gradient approximation (GGA). The muffin-tin radii are chosen as 2.0a.u. and 1.8a.u. for Sn and O (N), respectively. An energy cutoff of -7.0Ry is employed for the LAPW basis to describe the wave functions in the interstitial region, and potential and charge densities inside the atomic spheres are expanded in lattice harmonics up to $L=10$. We simulated $\mathrm{N}$-doped rutile $\mathrm{SnO}_{2}$ with a 72-atoms supercell, shown in Fig. 1a, where various substitutional nitrogen-doping levels are modeled by replacing one, two, three, and four oxygen atoms. This corresponds to stoichiometry of $\mathrm{SnN}_{x} \mathrm{O}_{2-x}$ with $\mathrm{x}=0.0208,0.0417,0.0625$, and 0.0833 , which is comparable to that used in the experiment [10-13]. Geometry optimization was done with the Brillouin zone sampling limited to the Monkhorst-Pack [15] grid with $4 \times 4 \times 4 \mathrm{k}$ points. The convergence threshold for the self-consistent energy error was set to $10^{-6} \mathrm{Ry}$, and atomic relaxations were carried out until all components of the residual forces were less than $10^{-3} \mathrm{Ry} / \mathrm{bohr}$.

The optimized 72-atom $\mathrm{SnO}_{2}$ supercell with one nitrogen dopant is shown in Fig. 1b. The $\mathrm{N}$ 
atom is coplanar bond to three Sn ions with two long and one short distance, i.e., $r_{1}=2.094 \AA$ and $r_{2}=2.082 \AA$, respectively, which slightly relaxes outward about $0.034 \AA$ and $0.018 \AA$ in comparison with the $\mathrm{Sn}-\mathrm{O}$ bond length of the pure $\mathrm{SnO}_{2}$ lattice, respectively. This structural deformation leads to the charge density redistributions in $\mathrm{N}$-doped $\mathrm{SnO}_{2}$. Therefore, the unpaired electrons are located around $\mathrm{N}$ perpendicular to $\mathrm{NSn}_{3}$ plane, and it occupied a hybrid $\mathrm{N} 2 \mathrm{p}_{\pi}$-Sn 5p orbital, as shown in Fig. 1c.

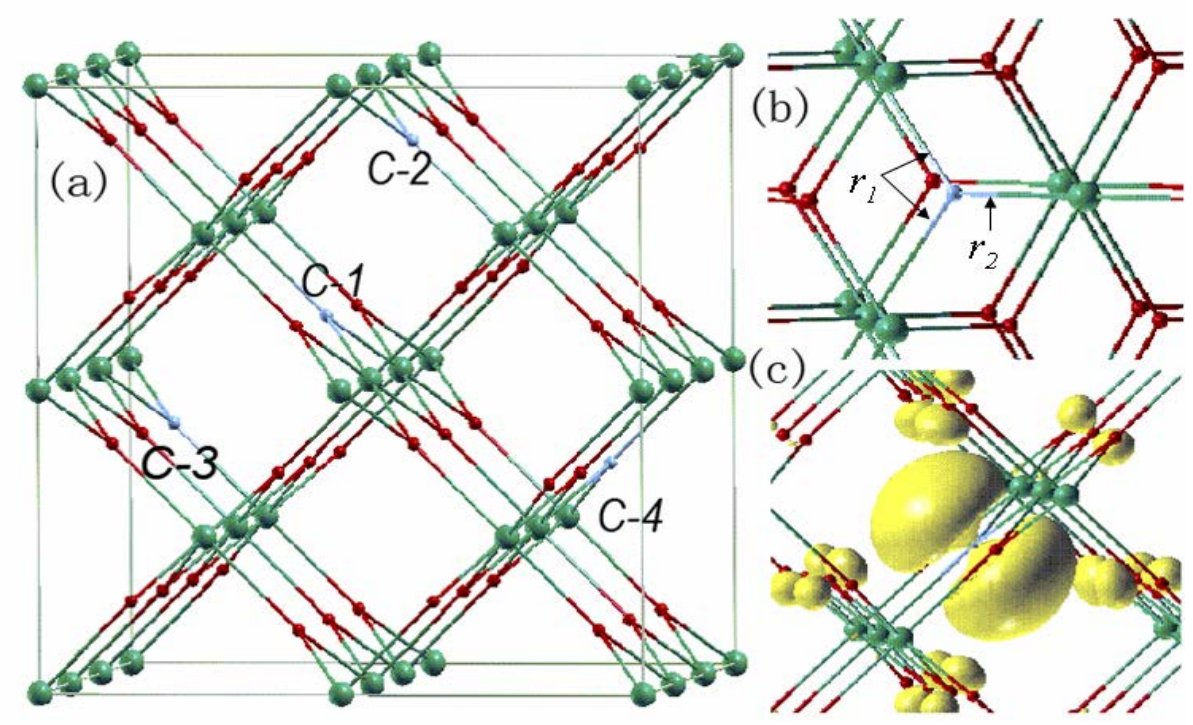

Fig. 1 The partial geometry of the $\mathrm{SnO}_{2}$ systems and spin density distribution. The big green spheres represent $\mathrm{Sn}$ atoms, the small red spheres represent $\mathrm{O}$ atoms, and the gray represents the $\mathrm{N}$ atom

\section{Results and Discussion}

\subsection{Electronic Structures}

To understand the effect of nitrogen on electronic properties, the band structure of pure $\mathrm{SnO}_{2}$ along the high symmetry directions of the Brillouin zone are calculated, shown in Fig. 2a. The band gap is about $2.1 \mathrm{eV}$ at highly symmetric $\Gamma$ point, which is less than the experimental value of $3.6 \mathrm{eV}$. The underestimated band gap can be due to the choice of GGA. In the uppermost valence band, $\mathrm{O} 2 p$ states are predominantly found between -8 and $0 \mathrm{eV}$, while $\mathrm{O}$ $2 s$ states appear in the range from -18 to $-15.5 \mathrm{eV}$ (not shown). The lowest conduction band is dominated by $\mathrm{Sn} 5 s$ states. The calculated electronic structures described in this work are consistent with the XPS spectrum data [16]. In Fig. 2b, one down-spin band gap state is occupied around $0.8 \mathrm{eV}$ when $\mathrm{N}$ atoms doped the $\mathrm{SnO}_{2}$. The band structure is mainly provided by $\mathrm{N} 2 \mathrm{p}$ states. The $\mathrm{N}$-doping $\mathrm{SnO}_{2}$ becomes magnetic semiconductor because the valence band and conduction band possess spin polarization. 


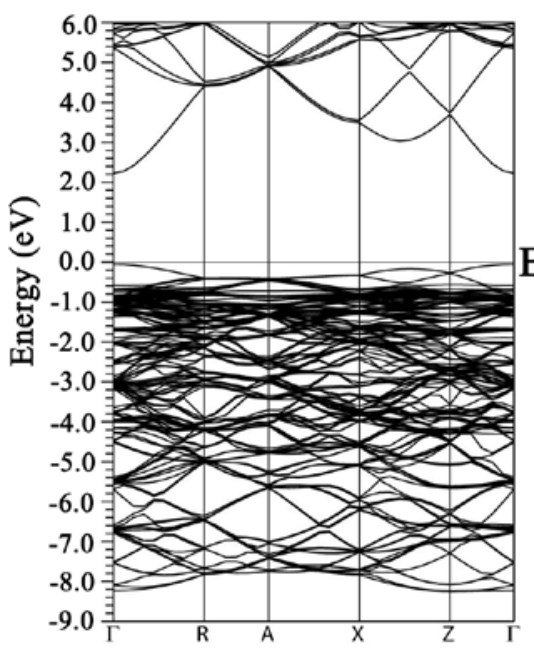

(a)

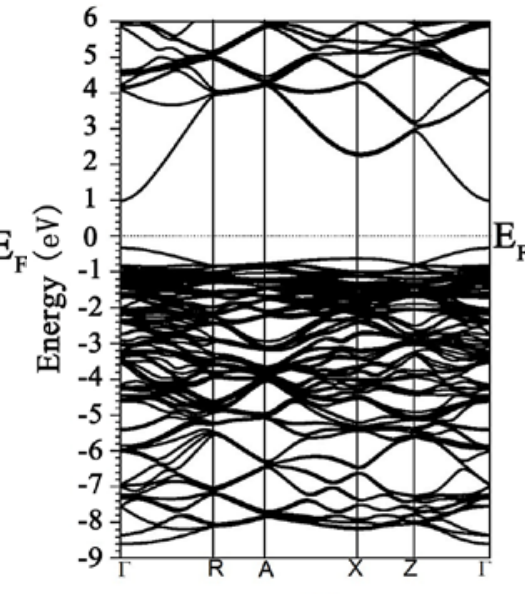

UP

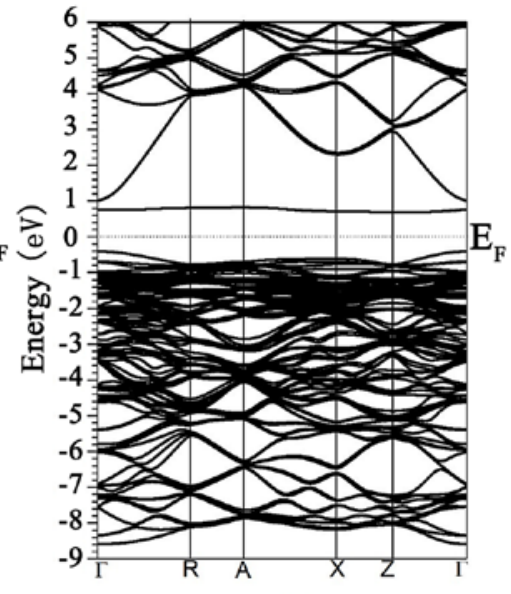

DN

(b)

Fig. 2 (a) The calculated band structure for pure supercell SnO2 (b) The band structure for $\mathrm{N}$-doping $\mathrm{SnO} 2$

Fig. 3 presents the calculated density of states (DOS) of $\mathrm{SnN}_{x} \mathrm{O}_{2-x}$ as a function of N-doping levels. Compared with the pure $\mathrm{SnO}_{2}$ in Fig. 2a, the band gap is obviously reduced, which is due mainly to the changes in the cell parameters. The N-doping introduces some occupied valence states below $E_{F}$, hybridized with $\mathrm{O} 2 p$ states, while only one down-spin band gap state is unoccupied. Therefore there is a local magnetic moment of $1.0 \mu_{\mathrm{B}}$ per nitrogen as shown in Fig. 1c. The spin-polarized electronic structure characteristic is in agreement with experiments for $\mathrm{N}$-doped $\mathrm{SnO}_{2}$ [12-13], in which the positions of gap states are depicted accurately. For the system doped with one $\mathrm{N}$ atom, it can be seen from Fig. $3 \mathrm{~b}$ that $\mathrm{N} 2 p$ localized states are formed in the energy gap close to conduction band, and the $\mathrm{N}$ localized states lead to a reduction of the energy gap of about $0.35 \mathrm{eV}$, which should be responsible for the red shift observed in the experiments [12]. With increasing nitrogen concentration shown in Fig. 4c-e, the density of $\mathrm{N} 2 p$ states increases, and therefore there are more occupied $\mathrm{N} 2 p$ states in the energy gap. Most importantly, for $x=0.0625$, the $\mathrm{N} 2 p$ states begin to mix with the valence band edge of $\mathrm{SnO}_{2}$ and thus lead to an obvious band gap narrowing in the $\mathrm{N}$-doped supercell. However, these $\mathrm{N} 2 p$ localized states above the valence band shift little when the number of substitutional $\mathrm{N}$ atoms increases, which explains why they has little contribution to the further energy gap narrowing with an increase of the nitrogen concentration in N-doped $\mathrm{SnO}_{2}$. The band gaps of $\mathrm{SnN}_{x} \mathrm{O}_{2-x}$ are 2.1, 1.8, 1.6, and 1.6eV, which correspond to $x=0.0208$, $0.0417,0.0625$ and 0.0833 , respectively. All these variations arouse our great interesting to the optical properties of $\mathrm{SnN}_{x} \mathrm{O}_{2-x}$. 


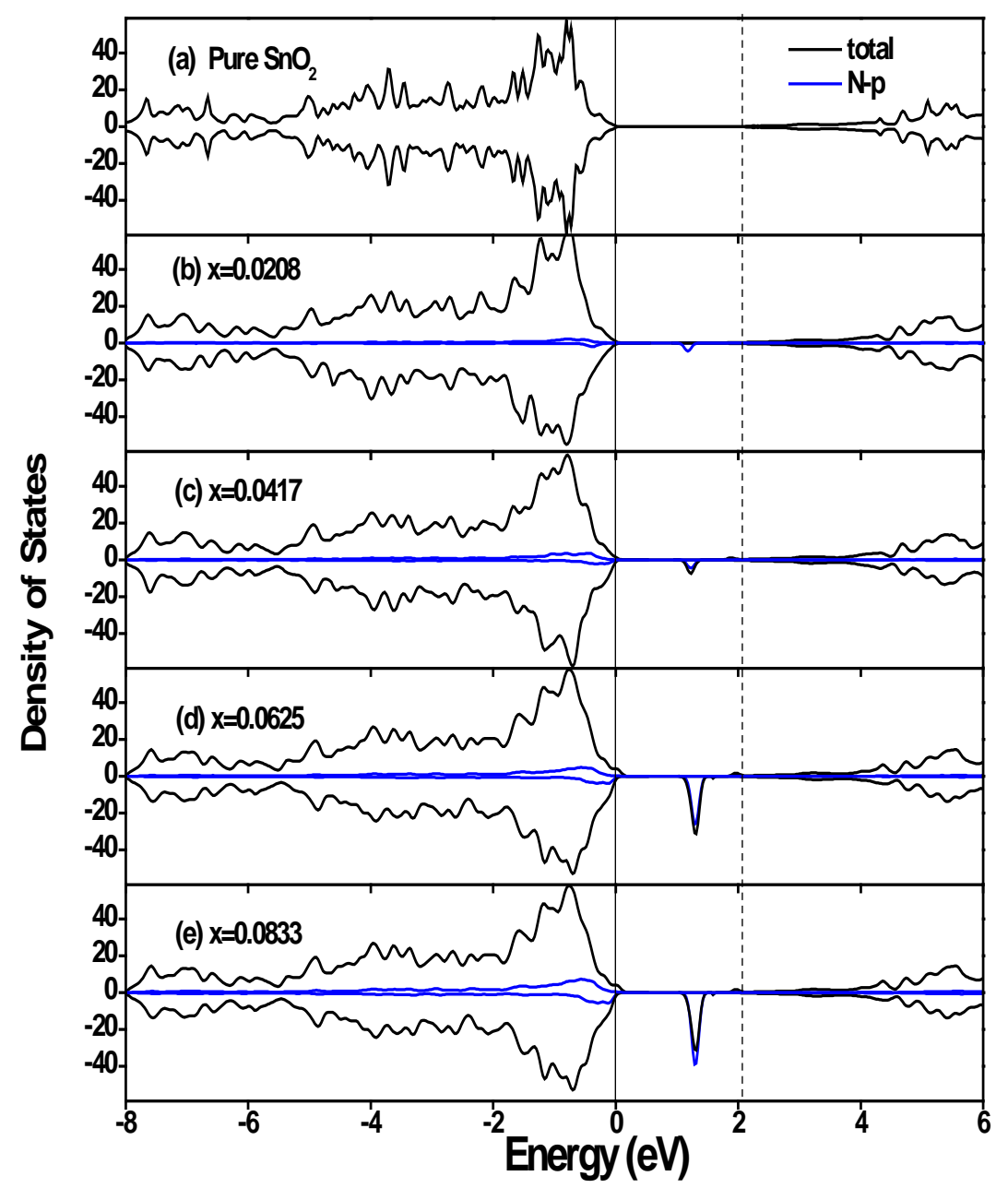

Fig. 3 The calculated density of states for the (a) pure 72-atom supercell, (b) doped 72-atom supercell with one $\mathrm{N}$ atom, (c) doped 72-atom supercell with two $\mathrm{N}$ atoms, (d) doped 72-atom supercell with three $\mathrm{N}$ atoms, and (e) doped 72-atom supercell with four $\mathrm{N}$ atoms, respectively.

\subsection{Optical Properties}

The optical absorption spectra under $1.5 \mathrm{eV}$ scissors operation with different levels of nitrogen doping were calculated, and the results are shown in Fig. 4. It is clearly observed that nitrogen incorporation induces the increasing optical absorption in visible range. The absorption peak has shifted to short wavelength range, which is in good agreement with of $E_{1}$ in Fig. 4. It indicates that the contribution of the visible absorption is originated from $E_{1}$ while not band gap narrowing. Optical absorption band edges shift slightly to the short wavelength range in the configuration of $x=0.0625$ and have no obvious shift in the other configurations. It is worth noting that the optical absorbance curves for the 6.25 atom \% nitrogen-doped $\mathrm{SnO}_{2}$ shows the highest photoresponse for visible light in the doped samples, which indicate that there is an optimum $\mathrm{N}$-doping level with higher photoresponse for visible light at low dopant concentrations. 


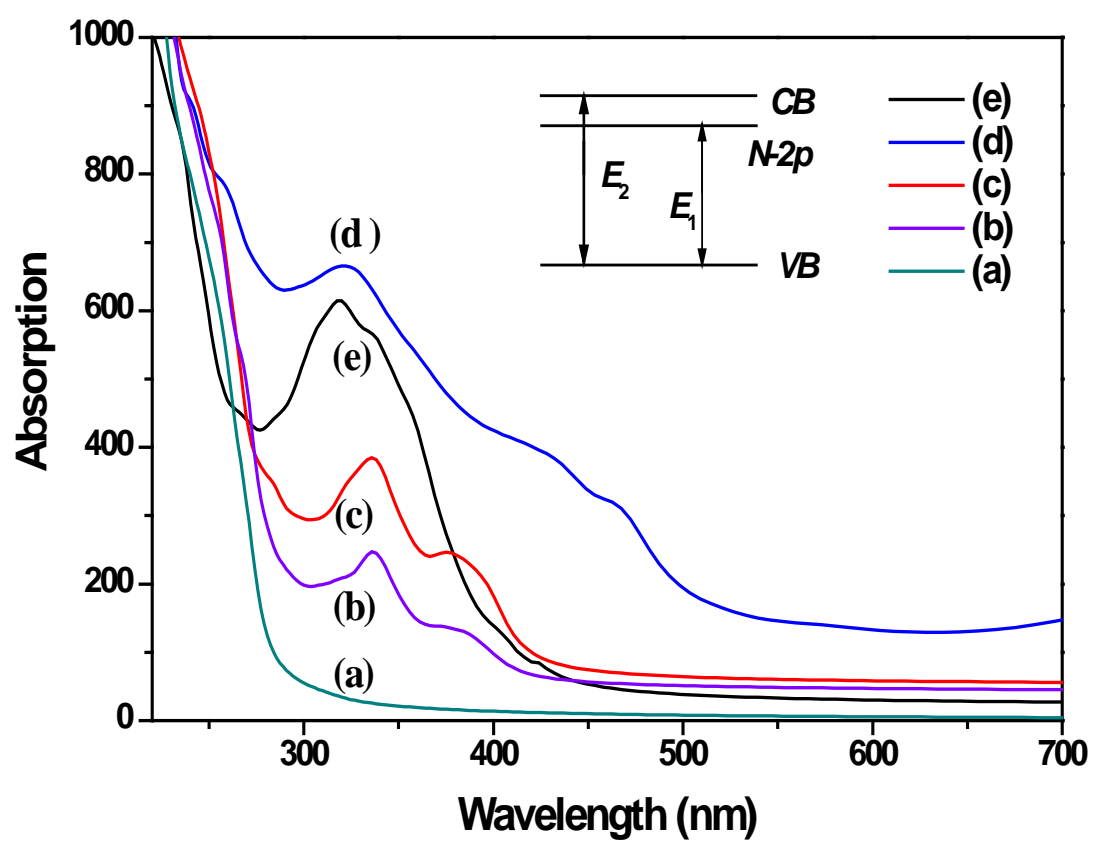

Fig. 4 Absorption spectra of $\mathrm{SnO}_{2}$ with different N-doping concentrations. (a) pure 72-atom supercell, (b) doped 72-atom supercell with one $\mathrm{N}$ atom, (c) doped 72-atom supercell with two $\mathrm{N}$ atoms, (d) doped 72-atom supercell with three $\mathrm{N}$ atoms, and (e) doped 72-atom supercell with four $\mathrm{N}$ atoms, respectively. Inset is the outline of the optical transition in

$$
\mathrm{SnN}_{x} \mathrm{O}_{2-x} \text {. }
$$

\section{Conclusion}

In summary, we carried out a first-principles study to investigate the electronic and optical properties of $\mathrm{SnN}_{x} \mathrm{O}_{2-x} . \mathrm{N}$ incorporation leads to the enhancement of $p$ states in the energy gap and the decrease of band gap. However, the band gap narrowing has no contribution to the enhancement of optical absorption in the visible range. The results indicate a possible optimum $\mathrm{N}$-doping level in $\mathrm{SnO}_{2}$ with a high visible photoresponse and as a photocatalytic material.

\section{References}

[1] M. Batzill, U. Diebold, The Surface and Science of Tin Oxide, Prog. Surf. Sci. 79 (2005) 47.

[2] S.S. Pan, C. Ye, X.M. Teng, H.T. Fan, G.H. Li, Preparation and characterization of nitrogen-incorporated $\mathrm{SnO}_{2}$ films, Appl. Phys. A, 85(2006), 21-24.

[3] A. Nuruddin, J.R. Abelson, Improved transparent conductive oxide $/ \mathrm{p}^{+} / \mathrm{i}$ junction in amorphous silicon solar cells by tailored hydrogen flux during growth. Thin Solid Films. 394 (2001) 48-62.

[4] R.G. Gordon, Criteria for Choosing Transparent Conductors. MRS Bull. 25 (2000) 52-57. 
[5] S. Tamura, T. Ishida, H. Magara, T. Mihara, O. Tabata, and T. Tatsuta, Transparent conductive tin oxide films by photochemical vapour deposition. Thin Solid Films 343-344 (1999) 142-144.

[6] C. Kilic, A. Zunger, Origins of Coexistence of Conductivity and Transparency in $\mathrm{SnO}_{2}$. Phys. ReV. Lett. 88 (2002) 095501.

[7] M.M. Bagheri-Mohagheghi, M. Shokooh-Saremi, The influence of Al doping on the electrical, optical and structural properties of $\mathrm{SnO}_{2}$ transparent conducting films deposited by the spray pyrolysis technique. J. Phys. D 37 (2004) 1248.

[8] Z. Ji, L. Zhao, Z. He, Q. Zhou, C. Chen, Transparent p-type conducting indium-doped $\mathrm{SnO}_{2}$ thin films deposited by spray pyrolysis. Mater. Lett. 60 (2006) 1387.

[9] M.M. Bagheri-Mohagheghi, M. Shokooh-Saremi, Electrical, optical and structural properties of Li-doped $\mathrm{SnO}_{2}$ transparent conducting films deposited by the spray pyrolysis technique: a carrier-type conversion study. Semicond. Sci. Technol. 19 (2004) 764.

[10] S.S. Pan, C. Ye, X.M. Teng, L. Li, G.H. Li, Localized exciton luminescence in nitrogen-incorporated $\mathrm{SnO}_{2}$ thin films. Appl. Phys. Lett. 89 (2006) 251911.

[11] S. S. Pan, Y. X. Zhang, X. M. Teng, G. H. Li, L. Li, Optical properties of nitrogen-doped $\mathrm{SnO}_{2}$ films: Effect of the electronegativity on refractive index and band gap. J. Appl. Phys. 103 (2008) 093103.

[12] S. Luo, P.K. Chu, Z. Di, M. Zhang, W. Liu, C. Lin, J. Fan, X. Wu, Vacuum electron field emission from $\mathrm{SnO}_{2}$ nanowhiskers annealed in $\mathrm{N}_{2}$ and $\mathrm{O}_{2}$ atmospheres. Appl. Phys. Lett. 88 (2006) 013109.

[13] R.Y. Korotkov, A.J.E. Farran, T. Culp, D. Russo, C. Roger, Transport properties of undoped and $\mathrm{NH}_{3}$-doped polycrystalline $\mathrm{SnO}_{2}$ with low background electron concentrations J. Appl. Phys. 96 (2004) 6445.

[14] K. Schwarz, P. Blaha, Solid state calculations using WIEN2K. Comput. Mater. Sci. 28 (2003) 259.

[15] H.J. Monkhorst, J. Pack, Special points for Brillouin-zone integrations. Phys. Rev. B, 13 (1976) 5188.

[16] J.M. Themlin, R. Sporken, J. Darville, R. Caudano, J.M. Gilles, R. L. Johnson, Resonant-photoemission study of $\mathrm{SnO}_{2}$ : Cationic origin of the defect band-gap states. Phys. Rev. B, 42 (1990) 11914. 